\title{
ATENÇÃO EM SAÚDE MENTAL: A PRÁTICA DO ENFERMEIRO E DO MÉDICO DO PROGRAMA SAÚDE DA FAMÍLIA DE CAUCAIA-CE
}

[Mental health: doctor and nursing practice in the family health program]

\author{
Adail Afrânio Marcelino do Nascimento* \\ Violante Augusta Batista Braga**
}

RESUMO: Este estudo tem por objetivo analisar a prática do enfermeiro e do médico do Programa Saúde da Família (PSF) quanto à atenção em saúde mental, na perspectiva da Reforma Psiquiátrica. Estudo descritivo, de natureza qualitativa, realizado com sete enfermeiros e cinco médicos no Município de Caucaia-CE. Os dados foram coletados mediante entrevista semiestruturada e observação livre, no período de maio a agosto de 2003. Depois de agrupados em categorias e sub-categorias, os resultados mostram que os enfermeiros e médicos do PSF desenvolvem suas ações dentro da lógica da programação em saúde, cumprindo o protocolo previsto. Observamos que sentem muita dificuldade de lidar adequadamente com as demandas de saúde mental da comunidade assistida, condição que se manifesta através de algumas respostas do grupo, tais como: o modo confuso como definem o que seja saúde mental; reconhecimento de inabilidade no manejo das demandas da comunidade; desconhecimento e pouca utilização dos recursos da comunidade para atuarem em ações preventivas; utilização restrita de uma escuta sensível e do relacionamento interpessoal. Por fim, foi possível apreendermos que as ações de saúde mental desenvolvidas pelo grupo pesquisado são orientadas pela prática da psiquiatria tradicional, onde a medicalização e o encaminhamento são os instrumentos de escolha. Esta condição não vai ao encontro o que prevê a

*Enfermeiro da Escola de Saúde Pública do Ceará-ESP-CE, Mestre em enfermagem-UFC, Especialista em Saúde da Família-ESP-CE.

**Professora Adjunta do Departamento de enfermagem-UFC
Reforma Psiquiátrica quando inclui as ações de saúde mental entre aquelas desenvolvidas na atenção primária, criando uma rede de assistência que tem por objetivo atender as demandas da comunidade.

PALAVRAS CHAVES: Saúde mental; Saúde da Família; Reforma Psiquiátrica.

\section{INTRODUÇÃO}

A Constituição brasileira de 1988 incorporou algumas das propostas da Reforma Sanitária, que contemplavam as reivindicações da sociedade. $O$ sistema de saúde começou a se delinear em novos perfis, procurando implementar políticas públicas intersetoriais que garantam a qualidade de vida de todos. Desse modo, testemunhamos grandes avanços na legislação da saúde, sem que o mesmo possa ser evidenciado na prática efetiva dos serviços de atenção à saúde da população.

Mudanças estruturais como essas, decorrem de amplos processos sociais e políticos, como o ocorrido na $8^{a}$ Conferência Nacional de Saúde (1986) e, no caso da saúde mental, o Movimento dos Trabalhadores de Saúde Mental (1987), que se tornaram marcos decisivos para elaboração das políticas públicas que se aproximam daquilo que a sociedade deseja para aquele momento histórico.

Por meio das mudanças surge a Reforma Psiquiátrica brasileira, como proposta à ruptura do modelo clínico-psiquiátrico, fazendo-se necessário à construção de novos saberes e práticas, num processo de desconstrução e reconstrução de 
novas formas de perceber a saúde mental e seus meios de intervenção, na busca de atender não mais a doença, mas a pessoa em sofrimento mental, procurando assisti-la nos vários níveis de atenção e em estruturas diversas, substitutivas do hospital psiquiátrico tradicional.

O processo de Reforma Psiquiátrica, ora em implantação no país, teve início na década de 1970, com as demandas do Movimento de Trabalhadores em Saúde Mental (movimento de profissionais da assistência psiquiátrica nas instituições públicas) e com o respaldo da "Declaração de Caracas", aprovada no dia 14 de novembro de 1990 pela Organização Panamericana de Saúde (OPAS) e da Organização Mundial de Saúde (OMS) (DESVIAT, 1999).

A trajetória da assistência em saúde mental, a princípio, revela uma prática psiquiátrica voltada para o tratamento hospitalar, desenvolvendo-se como um meio de exclusão do doente mental, ceifando-lhe o direito de ser cidadão e afastandoo da família e da sociedade (DALMOLIN, 2000).

A principal proposta reformista brasileira, na área de saúde mental, concretiza-se no Projeto de Lei 3.657/89, do deputado Paulo Delgado (PT/ $M G)$, o qual propunha regulamentação dos direitos do doente mental em relação ao tratamento e indicava a extinção progressiva dos manicômios públicos e privados e sua substituição por recursos extra-hospitalar (DELGADO, 1989). Esse Projeto de Lei traz elementos que contribuem para superar o modelo vigente, tendo por base à implantação de uma rede de serviços extra-hospitalares que atenda a alguns preceitos, destacando-se: atenção interdisciplinar, proibição da construção de novos hospitais psiquiátricos ou contratação de mais leitos; inserir leitos e criar unidades psiquiátricas em hospitais gerais; criar Centro de Atenção Psicossocial (CAPS), Núcleo de Atenção Psicossocial (NAPS), pensão protegida, lares abrigados; integrar a saúde mental a estratégias ou programas de saúde, movimentos sociais e instituições.

Atualmente a Reforma Psiquiátrica tem como uma das bases legais, o Projeto de Lei que dispõe sobre a proteção e os direitos das pessoas portadoras de transtornos mentais e redireciona o modelo assistencial em saúde, o qual foi aprovado e sancionado pelo Presidente da República em
2001, na forma da Lei Federal 10.216 de 06 de abril de 2001 (BRASIL, 2001).

A Lei supra citada refere que a pessoa em sofrimento mental deverá ser assistida por uma rede de atenção em saúde mental, em vários níveis de complexidade, abrangendo desde os serviços especializados até a inclusão de ações em programas de saúde geral. Desse modo, os serviços de atenção primária, como o Programa de Saúde da Família (PSF), constituem bases importantes dessa rede, tornando-se a porta de entrada para o sistema de saúde. Hoje, constitui um elo mais próximo à Unidade de Saúde, à família e comunidade (AMARAL, 2000).

Assim, consideramos de fundamental importância que os profissionais do PSF, enfermeiros e médicos, estejam sensibilizados para compreender o modelo de organização familiar, proporcionando uma assistência mais abrangente, oferecendo à família da pessoa em sofrimento mental o apoio necessário para que possa assumir o seu papel na sociedade.

A importância da reestruturação da rede básica de saúde e a habilitação dos profissionais de saúde são, no intuito de reduzir a demanda dos transtornos mentais, evitando reincidência da criseinternação-crise. Nesse sentido, as Unidades Básicas de Saúde da Família (UBASF) devem estar preparadas para garantir uma assistência de qualidade, adequada as demandas da comunidade, incluindo as referentes à saúde mental.

Neste contexto, é mister que os profissionais do PSF, principalmente enfermeiros e médicos, aprofundem seus conhecimentos técnicos e científicos acerca das práticas em saúde mental, pois estas colaboram no direcionamento da ação profissional, fundamentando a pesquisa e qualificando cada vez mais sua prática (CARRARO, 1998).

Desta forma, objetivamos com esse estudo analisar, na perspectiva da Reforma Psiquiátrica, a prática dos enfermeiros e médicos do PSF de Caucaia-CE na atenção em saúde mental, que certamente, constituirá uma contribuição importante para repensarmos uma forma de melhor utilização dos dispositivos públicos, na busca de uma assistência de qualidade a uma parcela grande da sociedade posta à margem do sistema, excluída de seus direitos de cidadão. 


\section{METODOLOGIA}

O Caminho metodológico que utilizamos para investigarmos esta realidade, teve como opção de trabalhar com dados do município de Caucaia-CE por termos atuado no mesmo enquanto enfermeiro do PSF. Fazem parte da nossa pesquisa, enfermeiros e médicos do PSF que atuam nas UBASFs localizadas na região praiana. Optamos por trabalhar com esses profissionais, pois são estes que, na equipe de PSF, assumem a maioria das ações assistenciais, na lógica da vigilância á saúde, atuando na promoção da saúde e nas práticas educativas.

Selecionamos uma amostragem, através de critérios, como: atuação no PSF no mínimo de seis meses nas UBASFs do litoral e em aceitar participar da pesquisa. Os sujeitos da pesquisa foram sete enfermeiros e cinco médicos. Incluímos cinco comunidades da região praiana que são atendidas pelo PSF com diferentes características socioeconômicas, onde a maioria das famílias tem melhores acessos a serviços, com algumas demandas de atendimentos psicológicos e emocionais.

O período de coleta de dados compreendeu os meses de maio a agosto de 2003. Para a coleta de dados empíricos dessa investigação, utilizamos como instrumentos de coleta de dados, a entrevista semi-estruturada com um roteiro previamente testado, a partir da realização de um teste piloto com dois enfermeiros e três médicos, a observação livre e diário de campo.

Utilizamos o método de Análise de Conteúdo de Bardin (1977), o qual foi desenvolvido com a expectativa de obtermos subsídios para a interpretação a partir das falas dos sujeitos da pesquisa.

Para o cumprimento dos objetivos desse estudo, utilizamos a Resolução 196, de 10 de outubro de 1996 (Diretrizes e Normas Reguladoras de pesquisas envolvendo Seres Humanos) do Conselho Nacional de Saúde, que tem mérito de dar ênfase aos compromissos éticos com os sujeitos da pesquisa.

\section{APRESENTANDO E ANALISANDO OS ACHADOS DO ESTUDO}

\subsection{CARACTERIZAÇÃO DOS SUJEITOS DO ESTUDO}

Os sujeitos desse estudo, em sua totalidade, são constituídos por enfermeiros do sexo feminino $71,4 \%$, e $80 \%$ dos médicos com sexo masculino, onde apresentaram a idade entre 21 e 50 anos $84,6 \%$, a maioria são casados $84,6 \%$ e em relação a formação profissional em nível de graduação somente para $37,5 \%$ dos enfermeiros, onde $57,1 \%$ são especialistas e $42,9 \%$ graduados; já os médicos apresentaram $40 \%$ com mestrado, $40 \%$ são especialistas e $20 \%$ são residentes.

Com relação ao tempo de formado os entrevistados possuíam de 1 a 5 anos de formados para $50 \%$ dos enfermeiros e $40 \%$ dos médicos, os demais apresentavam acima de cinco anos; a grande parte dos entrevistados, entre médicos e enfermeiros tem entre 1 a 4 anos de trabalho no PSF (92,3\%); em relação à situação empregatícia, $25 \%$ dos enfermeiros têm somente uma fonte empregatícia, tendo o restante do grupo mais de um emprego, já os médicos em sua maioria possuem média de dois empregos além do PSF; os salários são maiores para os médicos, variando de 14 salários ou mais (100\%), enquanto que para os enfermeiros variaram de 4 a 10 salários mínimos $(100 \%)$.

\subsection{A PRÁTICA DE SAÚdE MENTAL DOS ENFERMEIROS E MÉDICOS DO PSF: APREENSÃO DE UMA REALIDADE}

\subsubsection{Ações desenvolvidas pelos enfermeiros e médicos do PSF}

O Programa Saúde da Família baseia-se na lógica da estruturação de ações programáticas na rede básica de saúde do SUS, como diz Mendes Gonçalves (1994, p. 30):

A ação programática em saúde pode ser definida como uma proposição de organizar o trabalho em saúde fundamentada no ideal da integração sanitária, para o que busca inspirar-se em tecnologias de base epidemiológica tomando como ponto de partida a lógica de estruturação de programas de saúde. 
Observamos que as práticas dos enfermeiros e médicos do PSF, estão voltadas para as ações de programas ministeriais, tais como: ações ligadas á saúde da mulher, da criança, do idoso, do adolescente, do trabalhador, da saúde bucal, e entre outras. Essas ações de programação em saúde são freqüentes na descrição de suas práticas no PSF:

\begin{abstract}
"Atendimento que envolve gestantes, hipertensos, diabéticos, crianças, programas como tuberculose e hanseníase [...] atendimento a demanda livre, pediatria, puericultura, visitas domiciliares, [...] a gente segue um calendário semanal" (M 05).
\end{abstract}

Os enfermeiros e médicos demonstraram que na maioria das vezes, a forma de organização do atendimento no PSF, é baseada na queixa clínica, ou seja, as ações de caráter curativa são mais dominantes, até mesmo pela grande quantidade de demanda da comunidade que hoje procuram as UBASFs.

Esse tipo de atendimento tem por base 0 modelo clínico biomédico que privilegia a prática médica curativa e assistencialista, conforme destacado por Mendes (1996). Constatamos, de certa forma, que no PSF os enfermeiros assumem o atendimento da maioria das demandas de atenção primária, enquanto os médicos dedicam maior parte do seu tempo ao atendimento clínico.

Desse modo, sabemos que esses profissionais do PSF devem estar organizados em suas atividades de forma que todos assumam o compromisso de fazer ações de caráter curativo, preventivo e de reabilitação. Percebemos em alguns relatos dos entrevistados que apontam silenciosamente atividades com características do modelo biomédico, como a demanda livre de atendimento, o tratamento das doenças como foco principal, contradizendo, em algumas situações, a filosofia do PSF, no qual se recomenda um atendimento humanizado, preventivo, interdisciplinar, numa perspectiva de demanda organizada em sistema de agendamento de consultas.

De acordo com Brêda (2001) a prática desenvolvida pelos profissionais do PSF deve ser baseada em relações abertas, flexíveis e democráticas, destacando, em especial, o aconselhamento, o diálogo, as oficinas de expressão, o trabalho grupal, levando de fato os princípios do PSF, o qual devem contemplar como foco maior à atenção primária em saúde, e não somente para prevenir doenças, mas também para inventar e reinventar novas práticas em saúde, fazendo ainda que, o poder criativo em todas as direções, não se restrinja à monotonia de uma prática meramente instrumental.

Conseguimos perceber nas entrevistas, que a grande maioria dos enfermeiros do PSF realizam a educação em saúde, mesmo apresentando uma acentuada dedicação ao atendimento clínico, e além de serem os membros da equipe que mais realizam atividades administrativo-gerenciais, de capacitação, de supervisão em campo e de participação de reuniões com a comunidade. Enquanto os profissionais médicos em seus discursos poucos se manifestaram sobre a prática da educação em saúde em suas rotinas de trabalho, isso leva-nos a refletir a formação médica que permeia ainda o modelo biomédico, como foi citado anteriormente.

"Além de atender os programas estabelecidos, realizo palestras, levo a educação em saúde dentro da comunidade" (E6).

"A educação em saúde é a minha ferramenta de trabalho no PSF" (E5).

"Trabalhar com educação em saúde é de suma importância para o PSF, é uma forma de humanização para o atendimento” (E1).

A prática da educação em saúde discutida pelos enfermeiros do PSF, é vinculada ao paradigma preventivo, com a ideologia da atenção primária em saúde e da promoção da saúde, defendida pelas organizações internacionais de saúde e pelas instituições de ensino.

Entendemos que a educação em saúde é uma grande ferramenta no PSF, no entanto, faz necessário discutir, que esta não pode ser entendida apenas como cuidados pessoais para evitar doenças ou para se obter mudanças individuais relativas à falta de higiene, ignorância e não obediência a determinados preceitos da prática médica considerada como cuidados preventivos importantes e necessários para a promoção da saúde. 
PSF

Atender as demandas de saúde mental no PSF é de suma importância para levar de fato a nova proposta á assistência a saúde mental, dentro dos preceitos da Reforma Psiquiátrica, a qual permite a criação de novos saberes e práticas, formando redes em atenção a saúde mental.

Portanto, o PSF mostra-se como um importante instrumento nessa rede de atenção, uma vez que tem por objetivo assistir as famílias, procurando ver à pessoa como um todo, dentro do contexto familiar, responsabilizando-se pela promoção da saúde, proporcionando-lhe os devidos atendimento e encaminhamentos, quando necessários.

"Não deixamos a oportunidade de trabalhar a saúde mental, sempre chegam pessoas nervosas, irritadas e a gente sempre orienta, seja com qualquer problema" (E1).

"A clientela que atendemos, alguns moram sozinhos, não tem ninguém para conversar, nem familiares e acabam indo ao posto de saúde, para que sejam ouvidos" (E 04).

"Ás vezes atendemos pessoas com alguma ansiedade, depressão, irritadas, tudo isso devido aos fatores externos, como desemprego, a ociosidade, atividade pesqueira" (M 04).

Este enfoque da demanda á saúde mental identificada pelos enfermeiros e médicos do PSF em suas práticas são de suma relevância para a concretização de um novo modelo de atenção em saúde mental. Sendo este um momento de construção e reconstrução, a qual a saúde mental e a doença mental sejam discutidas e identificadas por esses profissionais de saúde não apenas nas unidades especializadas, mas também na UBASF e nos serviços comunitários.

Diante dessa realidade, a demanda de saúde mental deve ser identificada, tratada e acompanhada pela equipe de PSF, considerando que todas as ações básicas de saúde são asseguradas pelos princípios e diretrizes do SUS. Dessa forma, o PSF é um campo fértil para a concretização da nova forma de pensar/fazer saúde e trabalhar a saúde mental na comunidade.

Pesquisas realizadas em Municípios como Camaragibe - PE e Vale do Jequitinhonha - MG revelaram caminhos possíveis no processo de reorganização da atenção básica à saúde, no tocante, a inclusão da saúde mental no PSF, mesmo sem ter uma rede de serviços especializada que atendesse às demandas em saúde mental. (CABRAL et al., 2000).

As experiências desses municípios foram em torno dos pressupostos da orientação e organização dos serviços de saúde mental integrados ao modelo de atenção à saúde, a estimulação da saúde mental e às várias dimensões da vida educativa, política, econômica, social e psicológica na comunidade, promovendo ações conjuntas com o PSF e equipe de saúde mental. As estratégias utilizadas partem da base de planejamento das ações a serem realizadas, como: pesquisa e estudo de campo em área-piloto; oficinas de sensibilização das equipes do PSF; monitoração sistemática das ações e a elaboração de uma proposta de atenção à saúde mental.

Sampaio e Barroso (1997) dão destaque às ações de saúde mental a serem assumidas pelas equipes do PSF, ressaltando: o acompanhamento de psicóticos crônicos; a importância da coordenação de grupos de queixas difusas; as visitas domiciliares em sistema conjunto com o CAPS, para atendimentos de crise e o acompanhamento terapêutico-reabilitador; a prevenção e o combate à dependência de benzodiazepínicos; a efetivação de sistema de referência e contra-referência para identificar precocemente os casos de transtornos mentais nas áreas; atendimento das emergências psiquiátricas e aconselhamento psicológico.

Reconhecemos que as ações de saúde mental desenvolvida pelos enfermeiros e médicos do PSF de forma geral não apresentam estrutura adequada ao atendimento da demanda em saúde mental, levando-os a agir de forma intuitiva, não ocorrendo sistematização das ações, porque elas têm ocorrido de forma eventuais.

Desse modo, as ações de saúde mental precisam ser intencionais, entretanto, esses profissionais devem aprofundar seus conhecimentos e marcos conceituais acerca das práticas em saúde mental e direcionem suas ações qualificando cada vez mais esta prática profissional. 
3.2.3 Representações dos sujeitos sobre o processo saúde/doença mental

O Programa Saúde da Família lida com uma nova proposta de reorganização da saúde, buscando compreender, também, a dimensão social do cuidado. Dessa forma, é de suma importância estimular as equipes de saúde da família a refletirem e a desenvolverem ações em saúde, notadamente, a saúde mental. A especificidade das ações do PSF exige do profissional não só preparo técnico, como maturidade e compromisso ético para um desempenho de uma prática bem embasada e que atenda as necessidades das famílias.

Cerqueira (1999, p. 76) ressalta a importância de se acompanhar as mudanças quando afirma que:

\begin{abstract}
Formar-se das velhas práticas não é de todo mal, o problema está em não se ter quase a possibilidade de conhecer e trabalhar efetivamente nas novas práticas e em se pensar que esse antigo modo de operar é o suficiente para dar conta das demandas daqueles que paulatinamente, no decorrer de nossa formação, se tornarão nossos objetos de cuidados.
\end{abstract}

Desta forma, entendemos que a criatividade, a sensibilidade e a renovação do conhecimento são necessárias para os profissionais do PSF. Sabe-se que um dos caminhos para a humanização da assistência é a qualificação profissional, onde as velhas práticas precisam ser substituídas e reinventadas, no intuito de concretizar a mudança na ação desenvolvida, abrindo oportunidades e novos caminhos para uma assistência competente e humanizada.

A construção de novos caminhos, bem como a perspectiva de se terem novas práticas exigem a reconstrução de conceitos antigos e o traçar de novas trajetórias. Partindo-se do que já está posto é que conseguiremos avançar e fazer emergir o novo, o atual. Foi esse pensamento que buscamos apreender, nas falas do grupo pesquisado, sua representação sobre o processo saúde/doença mental.

Partindo-se de uma conceituação ou da representação que cada profissional tem do processo saúde/doença mental é possível identificarmos o modo como se colocam frente a essa problemática. A forma de perceber o sujeito ou a sua doença influencia diretamente nas ações desenvolvidas para atender a demanda apresentada.

Com base nesse pressuposto, procuramos identificar, junto ao grupo pesquisado, o significado da doença e saúde mental e quais os problemas de saúde mental mais freqüentes e como procuravam atender.

"Um doente que se enquadra dentro de um perfil descrito no diretório das doenças mentais, onde classificam as diversas doenças mentais, assim classifico como uma doença mental" (M5).

"É difícil essa pergunta, uma pessoa ansiosa, depressiva, não é um doente mental, são crises" (E5).

"O doente mental é aquela pessoa que tem um sofrimento, jamais mensurado [...] que não dá para medir, matematicamente com exames sintomáticos ou exames complementares, [...] são vários distúrbios patológicos difícil em diagnosticar, como depressão e esquizofrenia" (M02).

"Ninguém tem doença mental fixa, por ela estar relacionada com o estado da pessoa, por isso que a doença mental é de difícil diagnóstico" (M1).

"É difícil conceituar, porque é um conjunto de sutileza de sintomas que, às vezes, não conseguimos diferenciar a pessoa entre "normal e anormal" e que estão dentro da própria sociedade, caminhando igual a todas as outras pessoas" (E3).

Alguns dos enfermeiros e médicos demonstraram dificuldade em definir a doença mental, utilizando termos como distúrbios patológicos, emocionais, mudanças comportamentais, diferenças entre normal e anormal, critérios para classificação da deficiência mental e difícil diagnóstico.

O conceito de doença mental perpassa por vários pontos de vista. Na área médica, a doença é uma entidade nosológica que pode ser diagnosticada por meio dos elementos constituintes da síndrome patológica, a qual aponta um desvio dos tecidos orgânicos. Na área social, a doença é provocada por uma disfunção orgânica, que incapacita o individuo para desempenho efetivo dos papéis sociais. Nas instituições que compõem a sociedade civil, a doença mental é incluída como desordem mental, transformando o ator portador de agente considerado patológico em um personagem desviante, atípico e disfuncional dentro da família (D'INCAO, 1992). 
Ao conceituar a doença mental é necessário compreender que o processo saúde doença mental é multicausal, ou seja, são várias causas que fazem com que o individuo venha a desenvolver, em determinado momento de sua história, um transtorno mental, haja vista que é bastante complexa a sua definição, considerando os estereótipos e preconceitos que envolvem esse conceito.

Verificamos, por meio dos discursos, que a doença mental é vista como um distúrbio patológico, um distúrbio emocional, comportamental que prejudica as atividades consideradas normais ou efetivas. Trazem em seus conceitos nuanças racionalistas e tradicionalistas em relação à doença mental, apresentando características psíquicas e orgânicas, como por exemplo, as atividades manuais prejudicadas.

Os discursos dão indicativos de uma associação da doença mental com as psicopatologias, como as alterações psicológicas e emocionais. Entretanto, a compreensão da doença mental faz necessário o conhecimento do processo saúde doença mental em torno da doença mental, tendo em vista a importância dos fenômenos em diferentes variáveis, como: o psicológico, o cultural e o social. Dessa forma, ao conceituar a doença mental, devem ser levado em consideração as variáveis determinantes, para não rotulá-la, assumindo mil facetas no seu entendimento.

Realizamos ainda, um levantamento de uma série de problemas de saúde mental na comunidade atendida pelos enfermeiros e médicos pesquisados. Levando em consideração a importância de identificar e discutir os problemas de saúde mental, numa visão interdisciplinar, buscando a desmistificação da doença mental, implementando novas formas de atendimento na prática profissional como: o acolhimento, a escuta sensível desses problemas, compreendendo para o sofrimento, seja qual for a sua origem, o psíquico ou psicológico.

"O maior problema de saúde mental nesta área é o alcoolismo, em seguida são os transtornos de esquizofrenia e de ansiedade, pacientes dependentes de benzodiazepínicos e as depressões de maneira em geral" (M1).
"Aqui, são os tipos de depressão em pescadores" (M3).

"Primeiro lugar vem a depressão; as vezes, os pacientes vêm atrás da velha receita azul, ansiolítico, temos alguns pacientes esquizofrênicos" (M4).

"É o quadro de depressão; a paciente já chega dizendo que tem depressão, diz que já teve algumas crises, chega chorando, os outros são alcoólatras e usuários de drogas devido a região de turismo" (E2).

Os problemas de saúde mental apresentamse como uma realidade no cotidiano das atividades do PSF, daí a importância da inserção das ações em saúde mental na Unidade Básica de Saúde da Família (UBASF).

Desse modo, os enfermeiros e médicos do PSF podem ser promotores de saúde mental, uma vez que estão constantemente em contato com as famílias assistidas, esforçando-se para desenvolver novas propostas de atenção à saúde mental, levando um atendimento descentralizado, com base comunitária, contemplando os princípios da Reforma Sanitária, os quais são: conceito ampliado de saúde, qualidade de vida, integralidade, interdisciplinaridade, descentralização, acessibilidade, equidade e universalização.

Nesta reflexão que estamos fazendo acerca dos problemas de saúde mental é de suma importância, para compreensão do sofrimento psíquico e que seja discutido não apenas nas unidades especializadas, mas, também nas UBASFs. A partir dos conteúdos das falas, podemos destacar que a maioria dos problemas de saúde mental são as depressões, a ansiedade, a esquizofrenia e a dependência química, com o uso abusivo de álcool e outras drogas.

Fica patente na falas que o alcoolismo é considerado como um problema social, visto como uma toxicomania pela Organização Mundial da Saúde. Para Laranjeira e Pinsky (1998), as patologias associadas ao álcool estão entre os mais sérios problemas psiquiátricos, onde $50 \%$ das internações psiquiátricas do sexo masculino são por alcoolismo.

Corroborando com a discussão, Tavares; Beria e Lima (2001) relacionam o uso de drogas como um problema de saúde pública, o qual vem se destacando na adolescência. Estudo realizado 
em Pelotas-RS, com adolescentes matriculados em escolas públicas e particulares demonstram que as substâncias mais utilizadas por eles foram o álcool (86,8\%), o tabaco (41\%), a maconha (13, $9 \%)$, solvente $(11,6 \%)$, os ansiolíticos $(8 \%)$, os anfetamínicos $(4,3 \%)$ e a cocaína (3,2\%). Assim, observamos que os maiores índices de dependentes químicos são detectados nas UBASFs da região litorânea, contribuindo, talvez, com isso fato de ser área turística e apresentando elevado consumo de drogas ilícitas.

Outra patologia referida nos discursos é a esquizofrenia, considerada como a mais grave das doenças mentais, cuja fisiopatologia ainda está para ser esclarecida, podendo, ainda, se manifestar em indivíduos biologicamente vulneráveis. Para a OMS (1998) a esquizofrenia é caracterizada pela apresentação dos seguintes sinais e sintomas: eco do pensamento, delírios, alucinações, neologismos, comportamentos catatônicos, apatia marcante, pobreza de discurso e embotamento emocional.

Os transtornos depressivos também são citados nos discursos dos entrevistados, como a depressão e ansiedade, constituindo um problema de saúde pública, devido à alta prevalência e ao impacto psicossocial. Justificando esse pressuposto, Fleck (2002) relata em seu estudo que os pacientes com transtorno depressivo são freqüentadores assíduos dos serviços de atendimento primário, porém, a maioria deles não é diagnosticada como tal. A depressão causa a maior proporção de incapacidade, representando quase $12 \%$ do total de doenças neuropsiquiátricas (OMS, 2001).

Um aspecto relevante observado são atividades desenvolvida pela clientela assistida pelo $P S F$, sendo importante ressaltarmos a atividade pesqueira, haja vista, os pescadores passam vários dias no mar, podendo desenvolver problemas de saúde mental, devido sua atividade laboral, causando-Ihe estresse, ansiedades, depressões e tensões, além do uso de álcool ou outras drogas.

Com base nos depoimentos, percebemos que os enfermeiros e médicos do PSF apresentam dificuldades em lidar com os problemas de saúde mental, principalmente na forma de atendê-los, podendo às vezes comprometer a qualidade do atendimento.

\section{CONCLUSÃO}

Concluímos que os enfermeiros e médicos do PSF desenvolvem suas ações dentro da lógica da programação em saúde, cumprindo o protocolo previsto pelo Ministério da Saúde. Observamos que sentem muita dificuldade de lidar adequadamente com as demandas de saúde mental da comunidade assistida, condição que se manifesta através de algumas respostas do grupo, tais como: o modo confuso como definem o que seja saúde mental; uso do encaminhamento como uma das principais estratégias de ação; reconhecimento de inabilidade no manejo das demandas da comunidade; desconhecimento e pouca utilização dos recursos da comunidade para atuarem em ações preventivas; utilização restrita de uma escuta sensível e do relacionamento interpessoal; reconhecimento da falta de treinamento, insegurança e falta de experiência profissional para lidar com essas demandas.

Por fim, foi possível apreendermos que as ações de saúde mental desenvolvidas pelo grupo pesquisado são orientadas pela prática da psiquiatria tradicional, onde a medicalização e o encaminhamento são os instrumentos de escolha. Esta condição é o oposto do que prevê a Reforma Psiquiátrica quando inclui as ações de saúde mental entre aquelas desenvolvidas na atenção primária, criando uma rede de assistência que tem por objetivo atender as demandas da comunidade.

Nosso estudo identificou a necessidade dos enfermeiros e médicos reinventarem novas práticas em saúde mental, repensando seus conceitos, a fim de que seja introduzido o novo modelo previsto na Reforma Psiquiátrica, viabilizando resolutividade dos problemas decorrentes das demandas da comunidade assistida.

Percebemos que é necessário sensibilizar e qualificar os enfermeiros e médicos do PSF para a atuação na área de saúde mental, a fim de evitar o uso rotineiro de práticas psiquiátricas tradicionais, atendendo às demandas em saúde mental na UBASF, evitando internações psiquiátricas desnecessárias.

Ficou evidente, a importância da inserção da saúde mental na atenção primária, mesmo considerando que este estudo, trabalha somente com algumas equipes de PSF, consideramos que 
ele atingiu os seus objetivos ao buscar uma maior aproximação com a realidade vivenciada pelos enfermeiros e médicos pesquisados, servindo de contribuição para uma reflexão mais ampla sobre a prática profissional que vem sendo desenvolvida no município.

ABSTRACT: This study objectifies to analyze nursing and doctor practice in the Family Health Program (abbreviated PSF in Portuguese) regarding mental health under the perspective of the Psychiatric Reform. It is as descriptive study, qualitave in nature, accomplished with seven nurses and five doctors of Caucaia municipality in Ceara State/Brazil. Data were collected through a semistructured interview and free observation from May to July/2003. After being grouped in categories and sub-categories, the results show that nurses and doctors of PSF carry out their actions according in logic of the programming in heath, accomplishing the foreseen protocol. We observed that they feel a lot of difficulty for working appropriately with the demands of the attended community's mental health, condition that become evident through some answers of the group, such as: the confused way that they define what is mental health; the use of the guiding as one of the main action strategies; inability recognition in the management of the community's demands; ignorance and low use of the management of the community's resources to act in preventive actions; restricted use of a sensitive listening and of the interpersonal relationship. Finally, it was possible to understand that the actions of mental health developed by the researched group are guided by the practice of the traditional psychiatry, in which the medicating and the guiding are the choice instruments. This condition not goes against that what the Psychiatric Reform foresees when it includes the actions of mental health among those developed in the primary attencion, creating a net pf attendance that has for objective to assist the community's demands.

KEY-WORDS: Mental Health; Family Health; Psychiatric Reform.

\section{REFERÊNCIAS}

AMARAL, M. Atenção à saúde mental na rede básica: estudo sobre a eficácia do modelo assistencial. Rev. Saúde Pública, São Paulo, v. 31, n. 3, 2000.

BARDIN, L. Análise de conteúdo. Lisboa: Edições 70, 1977.

BRÊDA, M. Z. O cuidado ao portador de transtorno psíquico na atenção básica de saúde. Dissertação (Mestrado) - Centro de Pesquisa Aggeu Magalhães, Fundação Oswaldo Cruz, 2001.

CABRAL, B. et al. (Org.). Saúde loucura: saúde mental e saúde da família. São Paulo: Hucitec, 2000. p. 137-152.

CARRARO, T. E. Marco conceitual: subsídio para a assistência de enfermagem. Cogitare Enfermagem, Curitiba, v. 3, n. 2, p. 105-108, 1998.

CERQUEIRA, P. Ensino e assistência em saúde mental. Cad. IPUB, Rio de Janeiro, n.3, p. 75-83, 1999.

DALMOLIN, B. M. Trajetória da saúde mental no Brasil: da exclusão a um novo modelo. Mundo Saúde, São Paulo, v. 24, n. 1, p. 51-58, 2000.

DELGADO, P. Projeto de lei No 3657/89. Brasília, DF: Senado Federal, Centro Gráfico, set.1989.

DESVIAT, M. A reforma psiquiátrica. Rio de Janeiro: Fiocruz, 1999.

D'INCAO, M. A. A doença mental e a sociedade: uma discussão interdisciplinar. Rio de Janeiro: Graal, 1992.

FLECK, M. P. A. et al. Associação entre sintomas depressivos e funcionamento social em cuidados primários à saúde. Rev. Saúde Pública, São Paulo, v.36, n. 4, p. 431-438, 2002.

LARANJEIRA, R; PINSKY, I. O alcoolismo. São Paulo: Contexto, 1998.

MENDES, E. V. (Org.). Uma agenda para a saúde. São Paulo: Hucitec, 1996. 
MENDES GONÇALVES, R. B. Tecnologia e Organização Social das Práticas de Saúde. São Paulo: Hucite/Abrasco, 1994.

OMS. Cid-10 - Critérios diagnósticos para pesquisa. Porto Alegre: Artes Médicas, 1998.

ORGANIZAÇÃO PAN-AMERICANA DE SAÚDE/ ORGANIZAÇÃO MUNDIAL DE SAÚDE. Relatório sobre a saúde do mundo. Saúde mental: Nova concepção, Nova esperança. Brasília: Ed. Gráfica, 2001.

SAMPAIO, J. C.; BARROSO, C. M. C. Texto complementar para treinamento de profissionais de CAPS e PSF. Quixadá-Ce, 1997.

TAVARES, B. F.; BERIA, J. U.; LIMA, M. S. Prevalência do uso de drogas e desempenho escolar entre adolescentes. Rev. Saúde Pública, São Paulo, v. 35, n. 2, p.150-158, 2001. 